\title{
Yeast D-Amino Acid Oxidase: Structural Basis of its Catalytic Properties
}

\author{
Loredano Pollegioni ${ }^{1}$, Kay Diederichs ${ }^{2}$, Gianluca Molla ${ }^{1}$ \\ Stephan Umhau ${ }^{2}$, Wolfram Welte ${ }^{2}$, Sandro Ghisla ${ }^{2}$ and \\ Mirella S. Pilone ${ }^{1 *}$
}

\author{
${ }^{1}$ Department of Structural \\ and Functional Biology \\ University of Insubria \\ via J.H. Dunant, 3 \\ 21100 Varese, Italy \\ ${ }^{2}$ Section of Biology \\ University of Konstanz \\ P.O. Box 5560-M644 \\ D-78457 Konstanz, Germany
}

\begin{abstract}
The 3D structure of the flavoprotein D-amino acid oxidase (DAAO) from the yeast Rhodotorula gracilis (RgDAAO) in complex with the competitive inhibitor anthranilate was solved (resolution 1.9 A) and structural features relevant for the overall conformation and for catalytic activity are described. The FAD is bound in an elongated conformation in the core of the enzyme. Two anthranilate molecules are found within the active site cavity; one is located in a funnel forming the entrance, and the second is in contact with the flavin. The anchoring of the ligand carboxylate with Arg285 and Tyr223 is found for all complexes studied. However, while the active site group Tyr238-OH interacts with the carboxylate in the case of the substrate D-alanine, of $\mathrm{D}-\mathrm{CF}_{3}$-alanine, or of L-lactate, in the anthranilate complex the phenol group rotates around the $\mathrm{C} 2-\mathrm{C} 3$ bond thus opening the entrance of the active site, and interacts there with the second bound anthranilate. This movement serves in channeling substrate to the bottom of the active site, the locus of chemical catalysis. The absence in RgDAAO of the "lid" covering the active site, as found in mammalian DAAO, is interpreted as being at the origin of the differences in kinetic mechanism between the two enzymes. This lid has been proposed to regulate product dissociation in the latter, while the side-chain of Tyr238 might exert a similar role in RgDAAO. The more open active site architecture of RgDAAO is the origin of its much broader substrate specificity. The RgDAAO enzyme forms a homodimer with $C 2$ symmetry that is different from that reported for mammalian D-amino acid oxidase. This different mode of aggregation probably causes the differences in stability and tightness of FAD cofactor binding between the DAAOs from different sources.

(C) 2002 Elsevier Science Ltd. All rights reserved
\end{abstract}

Keywords: D-amino acid oxidase; crystal structure; anthranilate; dimerization mode; substrate access

\section{Introduction}

D-Amino acid oxidase (DAAO, EC 1.4.3.3) was one of the first flavoproteins to be discovered in the mid-30s of the last century ${ }^{1}$ and has played a

To their great sorrow, professor Vincent Massey died on August 262002 (S.G., M.S.P., L.P.). This paper is dedicated to his memory.

Abbreviations used: $\mathrm{CF}_{3}$-D-ala, 3,3,3-trifluoro-Dalanine; DAAO, D-amino acid oxidase; D-AAT, D-amino acid aminotransferase; GR, glutathione reductase; LAAO, L-amino acid oxidase; pkDAAO, pig kidney D-amino acid oxidase; RgDAAO, Rhodotorula gracilis D-amino acid oxidase.

E-mail address of the corresponding author: mirella.pilone@uninsubria.it prominent role in the development of present concepts in mechanistic flavin enzymology. It is a peroxisomal enzyme that catalyses the deamination of D-amino acid residues to their imino acid counterparts with concomitant reduction of FAD (see equation (1)). The reduced flavin is subsequently re-oxidized by molecular oxygen generating $\mathrm{H}_{2} \mathrm{O}_{2}$ (equation (2)), and the imino acid is released into solvent where it spontaneously hydrolyses to the corresponding $\alpha$-keto acid and ammonia (equation (3)):

$$
\begin{aligned}
& \mathrm{R}-\mathrm{CH}\left(-\mathrm{NH}_{3}^{+}\right)-\mathrm{COO}^{-}+\mathrm{DAAO}-\mathrm{FAD}_{\mathrm{ox}} \\
& \rightarrow \mathrm{R}-\mathrm{C}\left(=\mathrm{NH}_{2}^{+}\right)-\mathrm{COO}^{-}+\mathrm{DAAO}-\mathrm{FAD}_{\text {red }} \mathrm{H}^{-}+\mathrm{H}^{+}
\end{aligned}
$$




$$
\begin{gathered}
\text { DAAO-FAD }{ }_{\text {red }} \mathrm{H}^{-}+\mathrm{O}_{2}+\mathrm{H}^{+} \\
\rightarrow \text { DAAO-FAD }{ }_{\mathrm{ox}}+\mathrm{H}_{2} \mathrm{O}_{2} \\
\mathrm{R}-\mathrm{C}\left(=\mathrm{NH}_{2}^{+}\right)-\mathrm{COO}^{-}+\mathrm{H}_{2} \mathrm{O} \\
\rightarrow \mathrm{R}-\mathrm{C}(=\mathrm{O})-\mathrm{COO}^{-}+\mathrm{NH}_{4}^{+}
\end{gathered}
$$

In yeast cells, the flavoprotein DAAO is involved in primary metabolism where the efficiency of D-amino acid utilization is fundamental for cell growth. ${ }^{2,3}$ Conversely, in higher organisms, where its reaction occurs in selected organs, the physiological function is not entirely clear. In kidney it is probably involved in detoxification of exogenous D-amino acid residues, ${ }^{4}$ and good arguments for a role in neurotransmission regulation in brain have been advanced recently. ${ }^{5}$ DAAO also has considerable biotechnological importance for the enzymatic conversion of cephalosphorin C into 7-amino cephalosporanic acid. ${ }^{6}$

Yeast and mammalian DAAOs share features such as the basic catalytic mechanism. However, they differ in important aspects such as catalytic efficiency, substrate specificity, aggregation state, stability, kinetic mechanism, and mode and effectiveness of FAD binding. Thus, DAAO from the yeast Rhodotorula gracilis ( $\mathrm{RgDAAO})$ has a $k_{\text {cat }}$

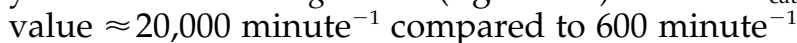
for pig kidney DAAO (pkDAAO) with D-alanine as substrate. ${ }^{7,8}$ This diversity arises mainly from a different rate-limiting step in the catalytic cycle. It is flavin reduction with $\operatorname{RgDAAO},{ }^{7}$ and product release with pkDAAO. ${ }^{8}$ Yeast DAAO can utilize large substrates such as cephalosporin C, this being only marginally the case with mammalian DAAO. ${ }^{9}$ While yeast DAAO exists in a stable dimeric state, pkDAAO dissociates easily and releases the FAD cofactor concomitant with loss of activity. The understanding of the molecular basis for these similarities and differences was the rationale for the present work.

In 1996, the 3D structure of pkDAAO at a $2.5-3.0 \AA$ resolution was reported. ${ }^{10,11}$ We have subsequently obtained the structure of the yeast enzyme, RgDAAO at $1.2-1.7 \AA$ resolution and have reported on some aspects of mechanistic significance. ${ }^{12}$ This, together with site-directed mutagenesis and mechanistic studies from our groups, ${ }^{13-16}$ has resulted in the conclusion that dehydrogenation by DAAO occurs via a hydride transfer. $^{12}$ The 3D structure of L-amino acid oxidase (LAAO) also has been reported recently, ${ }^{17}$ and this provides the basis for an understanding of the molecular factors leading to substrate D, or L-specificity.

Here we address the questions mentioned above pertaining to the similarities and differences between the yeast and mammalian DAAOs. In this context we present the crystal structure of RgDAAO in complex with the competitive inhibitor anthranilate (o-aminobenzoate). This molecule has attained mechanistic significance, since it was found to form the so-called "purple complexes" upon binding to pkDAAO ${ }^{9}$ and has been used widely as a model inhibitor. The structures of pkDAAO and LAAO in complex with anthranilate have also been solved; ${ }^{17,18}$ it was thus expected that comparisons of the modes of interaction of this ligand with the three enzymes would provide insights into similarities and differences of structure-function relationships of these flavoenzymes. We also address the factors that determine catalytic efficiency and the stability of the dimeric forms of DAAO.

\section{Results}

\section{Overall structure and topology}

The $\operatorname{RgDAAO}$ used here is a chimeric protein containing six additional residues (MARIRL) at the $\mathrm{N}$ terminus ${ }^{19}$ in addition to the 368 amino acid residues of the native form..$^{20}$ The 3D structure of the complex obtained in the presence of anthranilate is depicted in Figure 1. Secondary structure elements have been adapted and named according to the topology described by Mattevi et al. ${ }^{10}$ and some important elements are indicated. While there is correspondence between the FAD-binding domain (denoted with $\mathrm{F}$ ) for the RgDAAO and pkDAAO, the interface domains (denoted with I) are different, leading to a different mode of dimer formation (see below). For simplicity and ease of comparison we have nevertheless retained the original nomenclature ${ }^{10}$ for the interface domains. The secondary structure topology, consists of 11 $\alpha$-helices and $13 \beta$-strands; it is overall analogous to that of pkDAAO. ${ }^{10,11}$ The polypeptide chain has an irregular disposition and frequently crosses between the two domains whose main structural elements are central antiparallel $\beta$-sheets. Compared to pkDAAO, in the "head" region of RgDAAO there are three additional short $\alpha$-helices: based on the terminology used by Mattevi et al. ${ }^{10}$ these can be named $\alpha \mathrm{I}^{\prime}$ (between $\beta \mathrm{I} 1$ and $\beta \mathrm{I} 2$ ), $\alpha \mathrm{I}^{\prime}$ and $\alpha \mathrm{I}^{\prime \prime}$ (both after $\alpha \mathrm{I} 3$ ). Two main topological differences are also evident: the presence of a significantly shorter (six residues in RgDAAO versus 11 residues in pkDAAO) active site loop connecting $\beta \mathrm{I} 5$ and $\beta \mathrm{I} 6$ (Figure 1), and the presence of a long C-terminal loop (21 amino acid residues connecting $\beta F 5$ and $\beta F 6$ ), not present in other known DAAO sequences (see highlighted segments in Figures 1, 4 and 5). The functional effects of these structural differences will be discussed in detail below.

By structural overlay, the $\operatorname{RgDAAO}$ structure was compared to that of other flavoprotein oxidases (see Table 1). On the basis of structural and sequence homologies, ${ }^{20,21}$ RgDAAO can be classified as a member of the large glutathione reductase (GR) family (all the family members adopt the Rossmann fold ${ }^{22,23}$ ), and therein into the subgroup $\mathrm{GR}_{2}$ which was reported to show sequence similarity mainly within 30 residues in the $\mathrm{N}$-terminal 

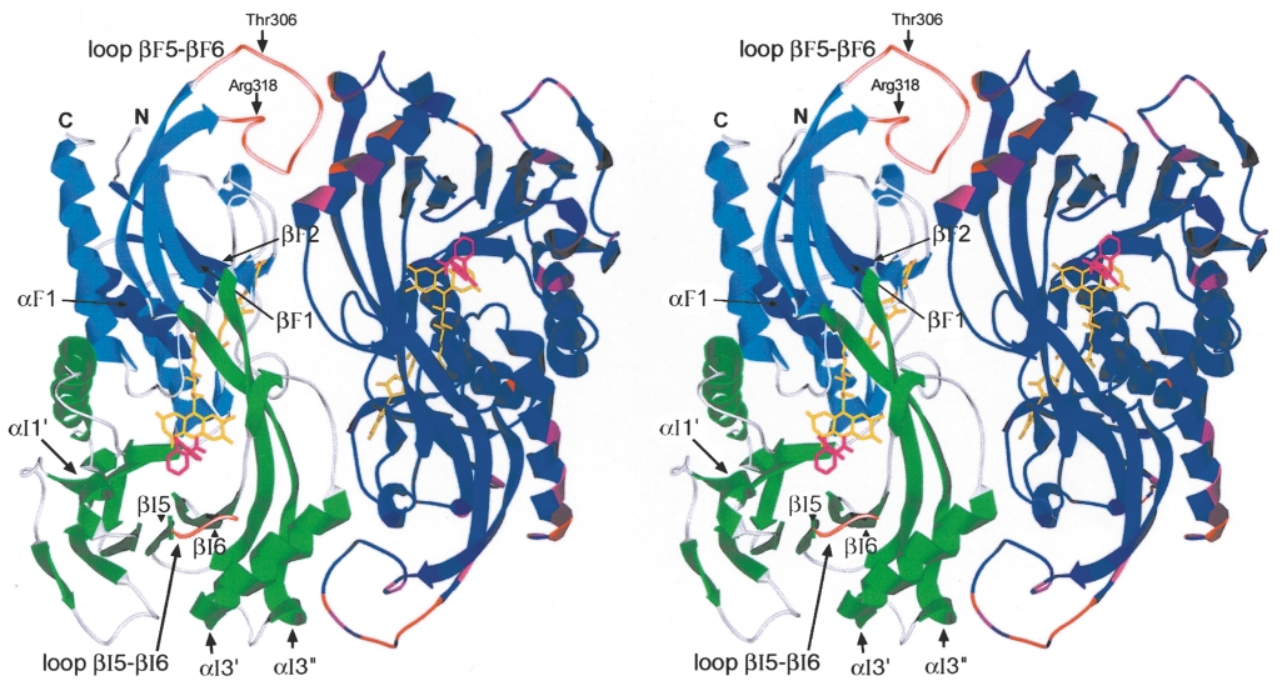

Figure 1. Ribbon representation of RgDAAO-anthranilate complex. The picture depicts the proposed dimer (see also Figure 3). In the monomer on the left-hand-side the "flavin-binding domain" is depicted in blue and the interface domain in green. The same terminology as introduced by Mattevi et al. for pkDAAO ${ }^{10}$ has been used in order to facilitate comparison between the two proteins. The segments in red denote two additional loops not present in pkDAAO; the $\beta F 1-\alpha F 1-\beta F 2$ secondary elements corresponding to the $\beta \alpha \beta$ motif of the Rossmann fold ${ }^{22}$ (the dinucleotidebinding motif ${ }^{5,26}$ ) are shown in dark blue. Arrows indicate the peptide bonds sensitive to proteolytic attack..$^{24,34}$ The carbon atoms of the FAD cofactor are in yellow and those of the two anthranilates inside the active site in magenta. The monomer on the right is represented using a color scaling for temperature factors: blue corresponds to a low $B$-factor and red to a high value.

region. ${ }^{23}$ Our superimposition procedure identifies significantly larger structurally homologous parts. However, in the case of the comparisons of $\mathrm{RgDAAO}$ and pkDAAO, the r.m.s. deviation of $1.38 \mathrm{~A}$ for 281 superimposable $\mathrm{C}^{\alpha}$ atoms (within a $3.5 \AA$ cut-off) is surprisingly high and reflects the evolutionary distance between the mammalian and the yeast enzyme. On the other hand, it is in accord with the relatively low percentage of identical residues $(31.8 \%)$ in the superimposed regions.

The closest structural relative of DAAO within the $\mathrm{GR}_{2}$ family is sarcosine oxidase. Indeed the two enzymes have identical topologies. In this case, the superimposed regions encompass sequence sections that are disposed over the whole sequence, whereas the other members of the $G_{2}$ family mainly superimpose in their $\mathrm{N}$-terminal region. ${ }^{23}$ No correlation between the r.m.s. deviation and the percentage of superimposed residues is evident from comparing
RgDAAO with other $\mathrm{GR}_{2}$ family members. This might hint at the large mutual evolutionary distance of members within this family, their broad range of substrate specificities and concomitant mechanistic peculiarities. High topological similarity is found within the $\mathrm{GR}_{2}$ family in the "flavin-binding domain" (Figure 1). In contrast only parts of the "interface domain" can be superimposed in pair-wise fashion.

\section{$\mathrm{N}$ and $\mathbf{C}$ termini}

At the $\mathrm{N}$ terminus only two (RL) out of six of the additional amino acid residues (MARIRL) can be modelled into the electron density, the remaining four apparently possessing a flexible conformation. This segment appears to be a significant component in crystal formation and growth, since we have failed to obtain crystals of wild-type (368 amino acid residues) protein. The backbone of residues Leu1 and Met1 (the original N-terminal

Table 1. Comparison of structural features of RgDAAO with other members of the $\mathrm{GR}_{2}$ family ${ }^{23}$

\begin{tabular}{lccc}
\hline $\begin{array}{l}\text { Protein } \\
\text { (PDB accession code) }\end{array}$ & $\begin{array}{c}\text { Number of residues } \\
\text { within } 3.5 \AA \text { A cutoff }\end{array}$ & $\begin{array}{c}\text { r.m.s. deviation of residues } \\
\text { within 3.5 A cutoff }\end{array}$ & $\begin{array}{c}\text { Sequence identity of residues } \\
\text { within 3.5 A cutoff (\%) }\end{array}$ \\
\hline pkDAAO (1aa8) & 281 & 1.38 & 31.8 \\
Sarcosine oxidase (1b3m_a) & 193 & 1.66 & 18.7 \\
Phenol hydroxylase (1foh) & 121 & 1.75 & 17.4 \\
p-Hydroxybenzoate hydroxylase (1bf3) & 120 & 1.65 & 19.2 \\
L-Amino acid oxidase (1f8r) & 120 & 1.87 & 24.4 \\
Cholesterol oxidase (1b4v) & 110 & 1.83 & 20.4 \\
Glucose oxidase (1cf3) & 108 & 1.47 & 18.7 \\
Polyamine oxidase (1b37_a) & 99 & 1.58 & 22.4 \\
Fumarate reductase (1d4c_a) & 97 & 26.8 &
\end{tabular}


residue of the wild-type protein) makes $\mathrm{H}$-bonds with two backbone residues Ala296 and Arg298 of the $\beta$-strand F5 of a symmetry-related protein molecule. Furthermore, the backbone carbonyl of His2 is H-bonded to Arg358, and Met1 is also in van der Waals contact with Val351 and Tyr359 of $\alpha$-helix $\alpha \mathrm{F} 5$ of the same symmetry-related molecule. Buried surfaces have been calculated using both the complete coordinates and a modelled protein lacking the additional $\mathrm{N}$-terminal sequence. In this latter case, the buried surface area between the monomers in crystal contact is decreased by $\sim 700 \AA^{2}$ in comparison to the value determined using the complete coordinates.

\section{Flexible parts}

In the long loop connecting $\beta \mathrm{F} 5$ and $\beta \mathrm{F} 6$ (Figure 1) the electron density for eight amino acid residues (Arg312-Gln319) is weak, indicating that part of the loop is very flexible. As was the case with pkDAAO, ${ }^{10,11}$ the C-terminal residues Ala362-Leu368 are also not visible in the electron density. This region contains the SKL tripeptide, corresponding to the PTS1 targeting sequence for peroxisomal proteins. This observation highlights the importance of the flexibility in the targeting signal for interaction with the PAS8-like receptor, the peroxisomal membrane protein that selectively binds the SKL peptide of peroxisomal proteins on the cytosolic face of peroxisomes. This part of the RgDAAO sequence is highly susceptible to proteolysis in vivo. The enzyme purified from $R$. gracilis cells consists of three isoforms when analysed by isoelectrofocusing. ${ }^{24}$ The microheterogeneity stems from the association of two polypeptide chains differing in the C-terminal tripeptide, giving rise to three different holoenzyme dimers. ${ }^{24}$

\section{FAD binding}

The FAD-binding patterns of $\operatorname{RgDAAO}$ and pkDAAO share an overall similarity while exhibiting some substantial differences. In both DAAOs the domain contains the conserved consensus Rossmann fold ${ }^{22} \beta \alpha \beta$ motif $(\beta F 1, \alpha F 1, \beta F 2)$, known as the dinucleotide-binding motif, ${ }^{25,26}$ common within FAD and $\mathrm{NAD}(\mathrm{P}) \mathrm{H}$-dependent oxidoreductases. ${ }^{23,27}$ The central part of this consensus motif is the sequence GXGXXG (Gly11, 13 and 16 of helix $\alpha \mathrm{F} 1)$ with the N-terminal end of helix $\alpha \mathrm{F} 1$ pointing toward the FAD pyrophosphate moiety. This serves in charge compensation, as observed for other dinucleotide-binding proteins. ${ }^{25}$ The residues interacting with the pyrophosphate oxygen atoms are more conserved compared to other segments, ${ }^{20}$ highlighting the importance of this interaction for cofactor recognition. ${ }^{20,23}$ The FAD in RgDAAO is found in an extended conformation typical of this GR family. ${ }^{23}$ The whole cofactor is buried inside the protein (Figure 1) and is not solvent accessible in agreement with previous deductions based on the absence of reac- tivity of enzyme reconstituted with modified FAD analogues with solvent-born reagents. ${ }^{28}$ The isoalloxazine ring is located at the interface of the two domains, with the re-side facing the inner part of the substrate-binding cavity. The large majority of the potential FAD H-bonds are with the protein, thus resulting in a tight net, as shown in Figure 2. The three rings of the isoalloxazine system form an almost perfect plane, wherein only the $\mathrm{C}(2)=\mathrm{O}$ and $\mathrm{C}(4)=\mathrm{O}$ groups occupy a slight "out-of-plane" position. As reported, ${ }^{12}$ the oxidized and reduced forms of the flavin do not differ significantly in their structural features and position. The flavin ring is held in position by a H-bond pair from its $\mathrm{N}(3) \mathrm{H}-\mathrm{C}(4)=\mathrm{O}$ to the Asn54 amide while $\mathrm{N}(5)$ is within $\mathrm{H}$-bond distance to Gly $52 \alpha-\mathrm{NH}$ (both residues are located on the loop region connecting $\beta$-strand F2 with $\alpha$-helix $\alpha \mathrm{I} 1)$. The flavin $\mathrm{N}(1)$ is within H-bond distance with Ser335 $(=\mathrm{O})$, such an interaction being absent in pkDAAO. The environment of the $\mathrm{O}(2)$ position is substantially different between $\operatorname{RgDAAO}$ and pkDAAO: in the latter the partial positive charge of a dipole induced by helix $\alpha \mathrm{F} 5$ is assumed to concur in the stabilization of the negative charges of the reduced flavin. Such a feature is absent in RgDAAO: $\mathrm{O}(2)$ forms two tight $\mathrm{H}-$ bonds with the backbone NH groups of Gln339 and Tyr338, both residues belonging to helix $\alpha \mathrm{F} 5$, while in pkDAAO $\mathrm{O}(2)$ interacts with a threonine ${ }^{10}$ (Figure 2). The hydrophobic part of the isoalloxazine ring makes van der Waals contacts with a pocket formed by the antiparallel $\beta$-strands I6 and I7 and interacts specifically with residues Ala51, Trp50, Gly183, Gly199, Thr201, Tyr223, and Arg285. The FAD diphosphate group forms H-bonds with Ala47, Ser48 and additionally with Ile15, the residue located at the beginning of the helix $\alpha \mathrm{F} 1$, and the helix dipole is assumed to contribute to the stabilization of the two negative charges of the diphosphate, as found with other members of the GR family. ${ }^{23}$ Four water molecules are found at optimal distance for $\mathrm{H}$-bond formation with three of the phosphate oxygen atoms (Figure 2). This is different from pkDAAO, where only three water molecules interact with two of the phosphate oxygen atoms. ${ }^{10,11}$ The highly conserved Asp34 of the mononucleotide-binding domain interacts via two strong $\mathrm{H}$-bridges with two $\mathrm{OH}$ groups of the AMP ribosyl moiety. One of these is also $\mathrm{H}$-bonded with the backbone $\mathrm{N}$ of Ser12, while the adenine moiety interacts with the backbone of Val162 and Arg35, and this gives the rationale of the observation that RgDAAO cannot productively use $\mathrm{FMN}^{28}$ (Figure 2). Thus, although different amino acid residues interact with AMP in yeast and the mammalian enzyme, the overall picture is similar in the two DAAOs.

\section{Mode of dimerization}

Native DAAO from R. gracilis is a stable $80 \mathrm{kDa}$ dimer of identical subunits, independent of the 


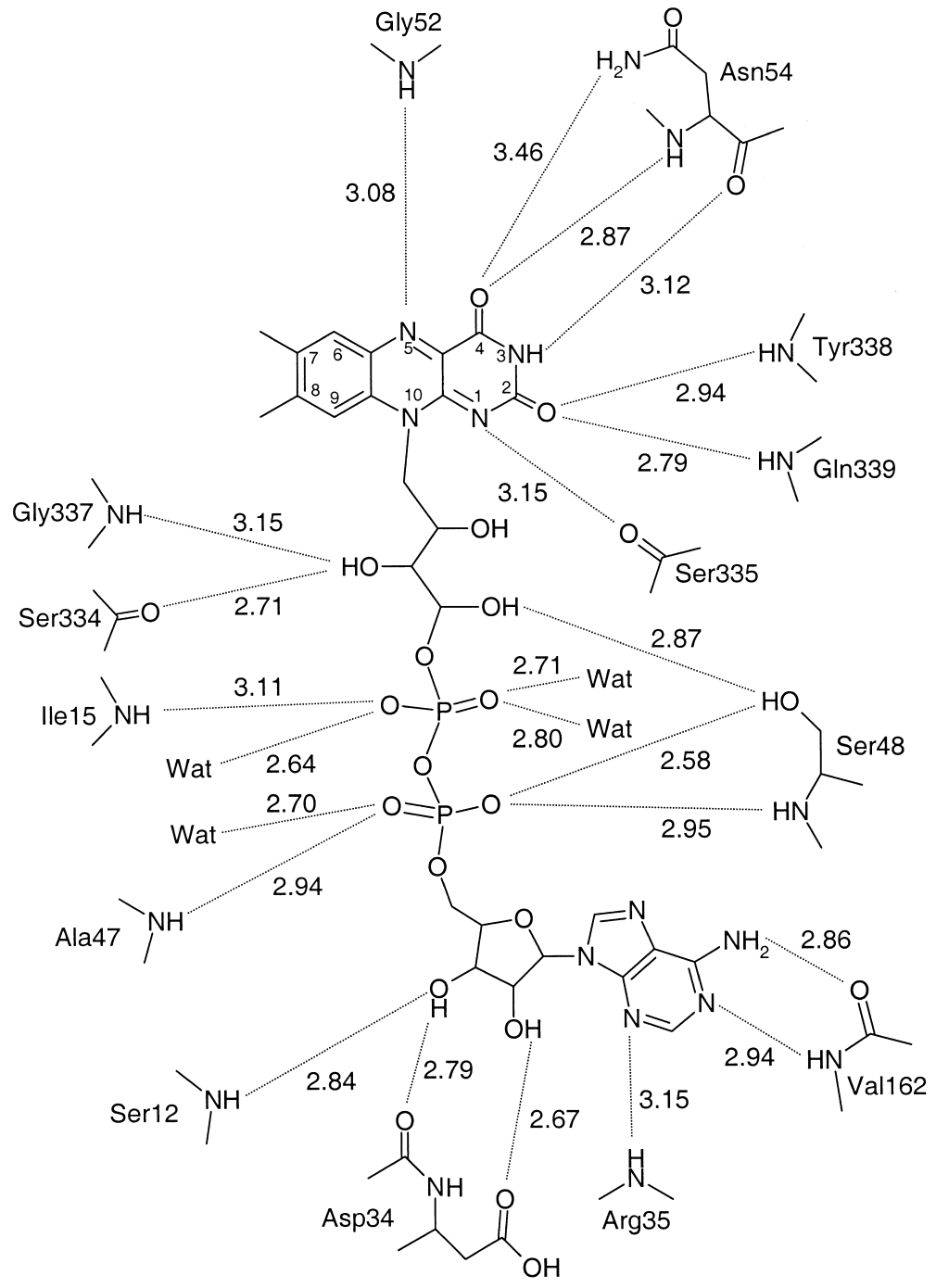

Figure 2. Schematic representation of the flavin-apoprotein interactions in RgDAAO. View on the si-face of the flavin. Residues interacting with donor or acceptor atoms of the cofactor are depicted. The hydrogen bonds are marked as dotted lines (distances are in $\AA$ ).

protein concentration. ${ }^{29}$ Each monomer contains one non-covalently bound FAD molecule. ${ }^{29}$ In contrast to this, the apoprotein form of RgDAAO is monomeric and rapidly converts to dimeric holoenzyme upon addition of FAD. ${ }^{30}$ Considering that the FAD cofactor is involved in many protein core contacts (see above), it is deduced that dimerization follows holoenzyme reconstitution. The dimer molecular mass estimated by dynamic light scattering is $\sim 79 \mathrm{kDa}$ and thus in accordance with a theoretical value of $82 \mathrm{kDa}$ for the recombinant $\operatorname{RgDAAO}^{19}$ This is consistent with RgDAAO forming a roughly spherical homodimer in solution. All crystals of RgDAAO have the tetragonal space group $I 422$ and approximate cell axes $a=b=121 \AA$ and $c=136 \AA$. The mode of dimerization cannot be derived directly from space group crystal symmetry under these circumstances.

In the 3D structure, the monomer of the asymmetric unit makes crystal contacts to give three symmetry-related dimers, indicating different, possible modes of monomer-monomer interaction (see Figures 1 and 3). For all the resulting potential dimers, the surface buried by the contact area was calculated using the CNS software package. ${ }^{31}$ For the "side to side" dimer, the buried surface area at the interface of two monomers was calculated as $678 \AA^{2}$ (Figure 3(a)). In analogy to the case of pkDAAO, also for RgDAAO a "head to head" mode of dimerization has to be discussed (compare Figure 3(b) and (c)). In this case the monomers would be related by a 2-fold axis with approximately $15 \%\left(1492 \AA^{2}\right)$ of the monomer accessible surface buried upon dimerization, yielding an elongated dimer of cylindrical shape $(112 \AA \times 45 \AA \times 31 \AA)$. This value is nearly the same as that reported for the pkDAAO dimer, where the buried surface area is $1512 \AA^{2}$ (Figure 3(c)). ${ }^{10}$ The largest buried surface $\left(3049 \AA^{2}\right)$ is, however, obtained for a "head to tail" monomer orientation with 2-fold symmetry (Figure 1) leading to 


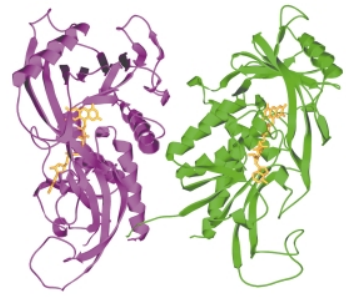

A) side to side

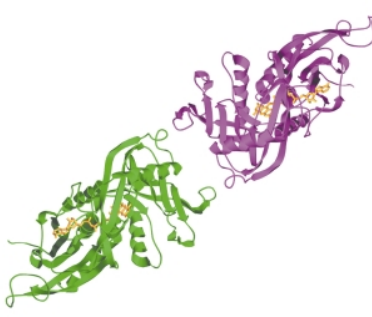

B) head to head

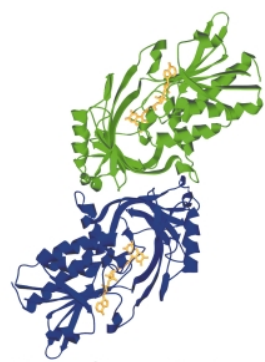

C) pkDAAO head to head

Figure 3. Possible, alternative modes of monomer-monomer interaction of DAAO from crystal contacts. (a) The "side to side" mode of dimer formation. This has the smallest buried surface area (see the text). (b) RgDAAO in the alternate head to head mode of dimerization. (c) The head to head mode of dimerization proposed for pkDAAO ${ }^{10,11}$ is shown for comparison. The head to tail mode of dimer formation proposed for RgDAAO is depicted in Figure 1.

the suggestion that this is the dimer form present in solution. Notably, loop $\beta F 5-\beta F 6$ of yeast DAAO is not conserved in other DAAO sequences. $^{20}$ The mean overall $B$-factors of RgDAAO are $\approx 15 \AA^{2}$, i.e. low (Figure 1). Only a few loop regions show slightly higher values such as residues 303-313 of the long loop connecting $\beta$ strands F5 and F6. However, the residues of this loop that are involved in crystal contact and con- tribute to dimer formation in the head to tail dimer have $B$-factors comparable to the mean.

\section{The active site cavity}

The active site of RgDAAO is a cavity delimited by the two long $\beta$-strands I4 and I8 bent around the isoalloxazine ring of the flavin and the two short $\beta$-strands I5 and I6 situated close to the (a)
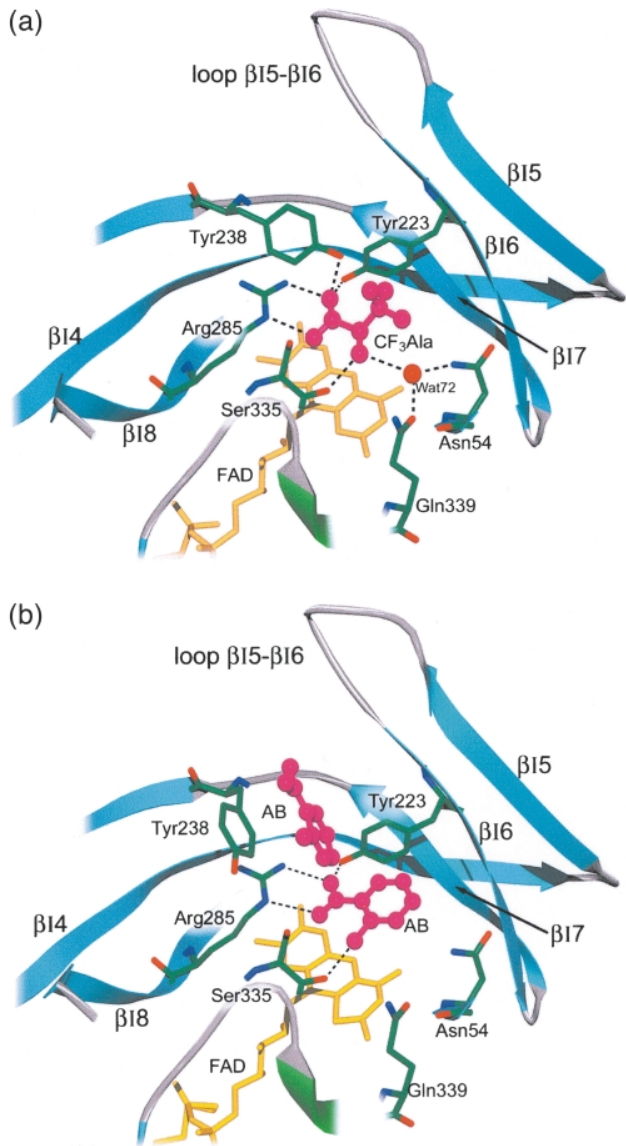
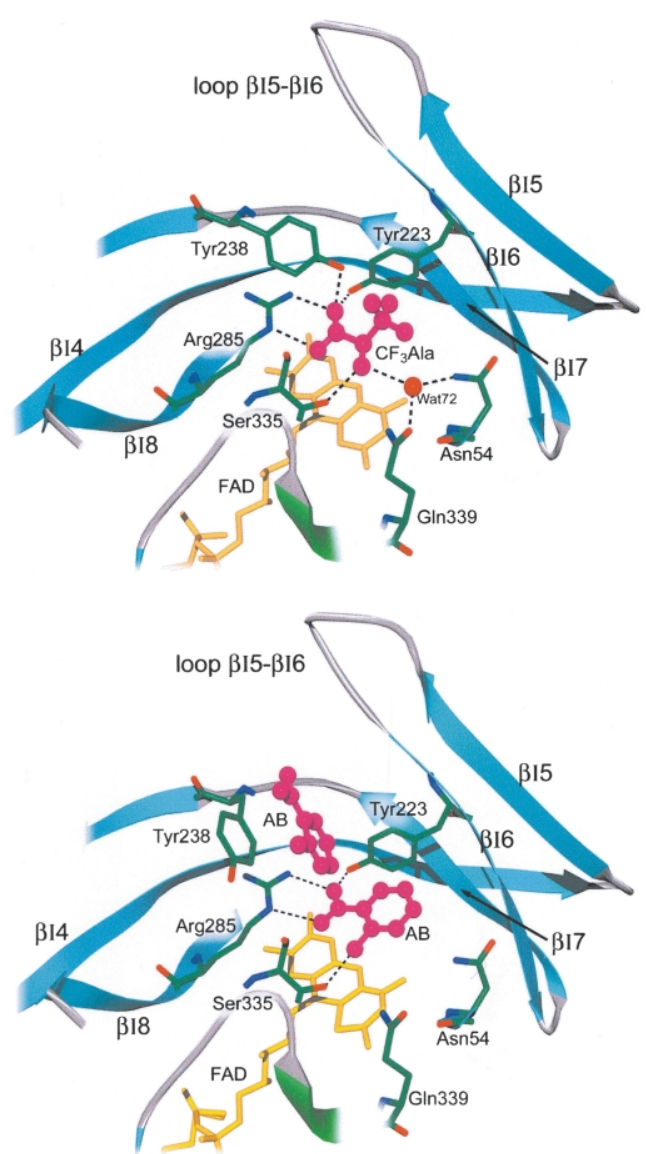

Figure 4. (a) Ligand-active site interactions of $\mathrm{RgDAAO}$. The ligand bound is $\mathrm{CF}_{3}-\mathrm{D}-\mathrm{Ala}$ (shown in magenta). Broken lines denote hydrogen bonds. Note the absence at the active site of functional groups that could participate in chemical events. (b) The active site region of RgDAAO in complex with anthranilate showing the channeling of two molecules of anthranilate (shown in magenta) into the active site cavity (Wat72 is no more present). 

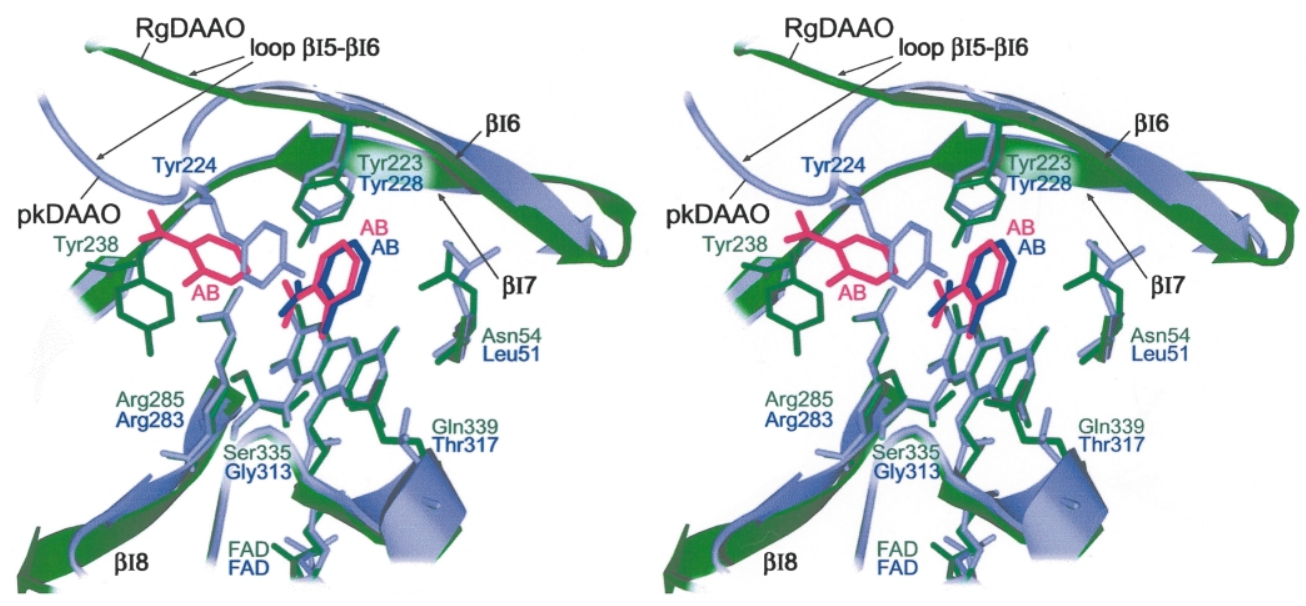

Figure 5. Comparison of the active site of RgDAAO and pkDAAO, both complexed with anthranilate. Features of the RgDAAO structure are in green, those of pkDAAO in light blue. The two molecules of anthranilate bound to RgDAAO are shown in magenta. The single anthranilate molecule bound to pkDAAO is in blue.

substrate-binding site (see Figure 4(a)). The flavin forms the "bottom" of the cavity. All these $\beta$-strands have an antiparallel orientation and are connected to each other by $\mathrm{H}$-bonds resulting in a rather rigid arrangement. At the re-side of the flavin, the loop connecting $\beta$-strand F2 and helix $\alpha \mathrm{I} 1$ is involved in backbone H-bonds with flavin functional groups (see above). On the si-side, the N-terminal end of the long helix $\alpha \mathrm{F} 5$ forms the boundary of the active site (see Figure 4). Interestingly the loop found in pkDAAO, which was proposed by Mattevi et al. to act as a "lid" controlling access to the active site ${ }^{32}$ is absent in RgDAAO (see below). This loop contains an important residue, Tyr224, that is probably involved in substrate/product fixation ${ }^{33}$ and interacts with the substrate $\alpha$-amino group and an active site water molecule. In RgDAAO the Tyr238 side-chain is placed at a similar position (Figures 4 and 5). Tyr238 is, however, located on a different segment of the chain, the loop connecting $\beta \mathrm{I} 7$ and $\alpha \mathrm{I} 3$.

The side-chains of the residues involved in substrate binding and fixation, including Wat72, retain the same positions in three of the complexes studied, $\mathrm{CF}_{3}$-D-Ala, L-lactate and D-alanine (Figure 4(a)). The interactions can be described as follows: (a) Arg285 forms a pair with the $\alpha-\mathrm{COO}^{-}$of the substrate, and both Tyr223-OH and Tyr238-OH interact with one of its oxygen atoms probably preventing rotation; (b) Ser335 $\alpha-\mathrm{NH}$ and Wat72 (held in position by Asn54 and Gln339) fix the ligand $\alpha \mathrm{NH}_{2} / \mathrm{OH}$ via a short and symmetric pair of H-bonds (both $2.72 \AA$ ); (c) the fourth, and frequently largest ligand $\alpha \mathrm{C}$ substituents, occupies the space provided by the active site cavity and extends towards its entrance. We have compared the substrate-binding modes by superimposing the 3D structures of RgDAAO (complexed with D-alanine or anthranilate), pkDAAO (with anthranilate) ${ }^{10,11}$ and $\mathrm{LAAO}^{17}$ (with anthranilate) while keeping the flavin moiety constant. The superposition of the active centre of pkDAAO and
RgDAAO in complex with anthranilate is shown in Figure 5 and will be discussed below.

\section{The anthranilate complex}

Two molecules of anthranilate are found at the active site in crystals soaked with this competitive inhibitor (Figures 1, 4(b) and 5). One is placed in the vicinity of the flavin $\mathrm{N}(5)$, at a position similar to that occupied by D-alanine, and the second is located at the entrance of the active centre cavity. Comparison of the binding mode of the first anthranilate with that of D-alanine, $\mathrm{CF}_{3}-\mathrm{D}-\mathrm{Ala}$, and L-lactate (Figures 4 and 5) shows that all of these ligands are located at similar positions, the H-bond interactions and conformation of Arg285, Tyr223 and Ser335 being essentially identical. From this it follows that the substrate $\alpha \mathrm{C}-\mathrm{H}$ is placed in the extension of the N(5) LUMO orbital of the flavin on its re-side. ${ }^{12}$ However, two significant differences are also evident: in the RgDAAO-anthranilate complex, Tyr238 has moved away from the ligand $-\mathrm{COO}^{-}$and it is too distant to make any discrete $\mathrm{H}$ contact(s) with the latter. Furthermore, the active site water W72, which forms an $\mathrm{H}$-bond with the $\mathrm{OH} / \mathrm{NH}_{2}$ function of the other ligands, is not found in the DAAO-anthranilate complex. The aromatic ring of anthranilate is in contact with the hydrophobic residues (Ile225, Phe58, Tyr223) within the binding pocket, possibly contributing to the overall binding enthalpy (anthranilate binding, $K_{\mathrm{d}} \approx 2 \mathrm{mM}$, is relatively tight compared e.g. to D-lactate, $16 \mathrm{mM}$ ). Displacement of Wat72 in the DAAO-anthranilate complex is due to the bulkiness of the ligand. The second anthranilate molecule lies parallel with, and in contact with, Tyr238, the latter having modified its position compared to that in the structures of the other complexes (Figures 4 and 5). The side-chains of Phe58, Ser215, Pro221, and the backbone of Ser234 and Ser235 form the entrance to the active site whose opening is 


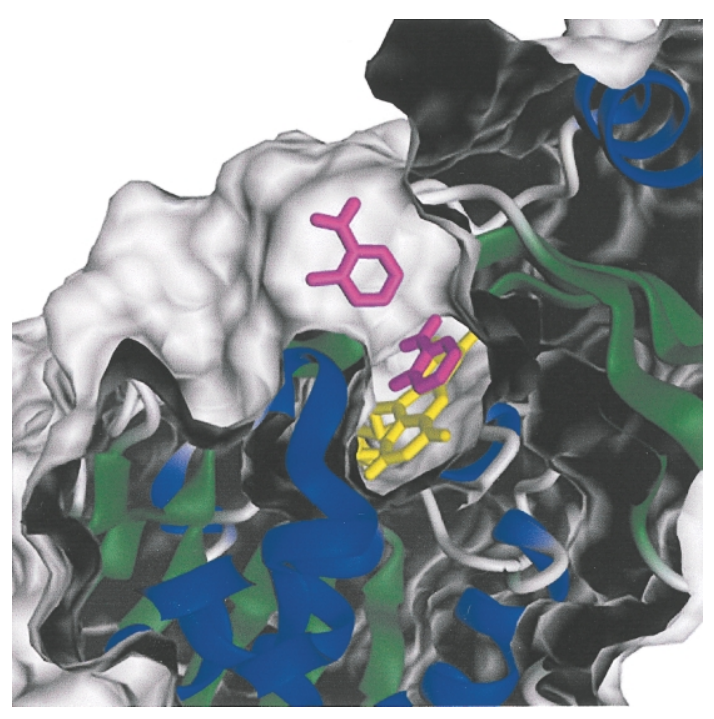

Figure 6. Molecular surface representation of the DAAO-anthranilate complex showing a magnified view of the funnel leading to the active site. The protein main-chain is shown as a coloured ribbon according to the domain colouring shown in Figure 1. The anthranilate and FAD molecules are depicted in magenta and yellow, respectively. One anthranilate molecule is bound into the active site in close contact with the flavin, whereas the plane of the second is parallel with that of the aromatic side-chain of Tyr238 (see also Figures 4(b) and 5). The surface map was produced with the program DINO†.

controlled by the orientation of the phenolic ring of Tyr238 (see above), and also provides a surface suitable to interact with non-polar and aromatic compounds (and substrates).

\section{Discussion}

On the basis of the data presented here, we propose that yeast DAAO dimerizes in a head to tail mode (see Figure 1 and compare with Figure 3 ) involving parts of loop 302-322 ( $\beta F 5-\beta F 6)$. This would be in line with results from limited proteolysis carried out with $\operatorname{RgDAAO},{ }^{24,34}$ which show that disruption of this loop abolishes the capacity to dimerize. The peptide bonds at Thr306 and Arg318 (Figure 1) of the mentioned loop are susceptible to trypsin, chymotrypsin and thermolysin proteolysis ${ }^{24,34}$ leading in all cases to active, monomeric holoenzyme containing two polypeptides of $\sim 34 \mathrm{kDa}$ (Met1-Arg305 or Met1-Leu312) and of $\sim 5 \mathrm{kDa}$ (Ala319-Ala362). ${ }^{24,34}$ This mode of dimerization yields a quasi-spherical particle and, due to the large contact area, should result in a stable dimeric protein. This is different from the head to head mode proposed for mammalian DAAO,${ }^{10}$ where the interaction is much weaker, and activity depends upon the aggregation state of the protein in solution. ${ }^{9}$

$\dagger$ http://www.biozentrum.unibas.ch/ xray/dino
Flavoenzymes are often equipped with flexible elements, which have a role in controlling the accessibility of the active site. ${ }^{35}$ In pkDAAO a loop, formed by residues 216-228, acts as a lid, which swings between an "open" and "closed" conformation to allow access/release of substrate/ product. $^{32}$ This conformational change is comparatively slow and controls the overall rate of turnover of the mammalian enzyme, where product release is rate-limiting. ${ }^{8}$ In yeast DAAO a corresponding loop that could exert the same function is not present; however, Tyr238 in a rudimentary fashion, appears to play a comparable role. The side-chain of this amino acid is assumed to take the open conformation in uncomplexed enzyme, and this might initiate an interaction with substrate, leading it into the bottom of the active site (Figure 6). Then, concomitantly with a conformational change positioning it further inside the active site, it contributes to the fixation of the substrate $-\mathrm{COO}^{-}$ in a position suitable for catalysis (see the different conformations of Tyr238 in Figure 4(a) and (b)). This is in agreement with the results obtained with RgDAAO Tyr238Phe and Tyr238Ser mutants, indicating that the main function of this group is in substrate/product exchange instead of in binding (the $K_{\mathrm{m}}$ value for the D-amino acid, as well the $K_{\mathrm{d}}$ value for ligand binding, are only slightly altered by the substitutions) (L.P., unpublished results).

A salient feature of the active site pocket is the presence of a wide cavity where the side-chain of the substrate can be accommodated (Figures 4 and 6). This provides a rationale for the preference of RgDAAO for D-amino acid residues carrying an aromatic and/or hydrophobic side-chain, compared to polar ones, ${ }^{4,9}$ and for its capacity to accommodate large residues, as in the case of cephalosporins. ${ }^{6}$ It also explains the reluctance of $\mathrm{RgDAAO}$ to act on amino acid residues carrying a polar and, in particular, a charged side-chain. The use of a single side-chain to control the accessibility of the active site has been observed also in UDP-Nacetylenolpyruvoyl-glucosamine reductase. ${ }^{36}$ In analogy to LAAO, where three anthranilate molecules are present in each protomer inside the channel leading to the active site ${ }^{17}$ the position of the two anthranilate molecules found in $\mathrm{RgDAAO}$ is taken to reflect the trajectory of the substrate from the surface to the locus of the catalytic event (Figures 4 and 6). The one anthranilate in contact with the flavin occupies the position assumed by normal D-amino acid substrates. The second one is in contact with Tyr238, and its amino group is positioned directly above the tyrosine ring. It is tempting to hypothesize that D-amino acid residues entering the active site behave similarly: their cationic ammonium group would undergo a cation- $\pi$-interaction ${ }^{37}$ and this could contribute substantially to the binding energy. Upon moving to the second binding site at the bottom of the active centre, depending on the $\mathrm{pH}$ the ammonium group would deprotonate ${ }^{12}$ in order to undergo 
catalysis. Such a $\pi$-interaction might be absent or reduced in the product, since the imino/immonium group readily hydrolyses (see equation (3)). After catalysis, and if imino acid hydrolysis occurs prior to dissociation, the product cannot be retained by $\pi$-interaction and would thus freely diffuse away. If this hypothesis holds, the "second" substrate site at the entrance of the cavity would act like a valve. The observed rotation of the Tyr238 side-chain would simply be effected by an induced fit mechanism, which optimally positions substrate and tyrosine ring with respect to each other.

An interesting feature of DAAOs and of flavoprotein oxidases in general is their ability to stabilize a negative charge on the flavin where it is localized mainly in the pyrimidine ring. This is the case, e.g. upon reduction (see equation (1)), with semiquinones, ${ }^{29}$ with modified flavins, ${ }^{28}$ and also in the case of sulphite binding. ${ }^{9,27}$ This stabilization translates into an increase in redox potential, a feature of most flavoprotein oxidases. ${ }^{38}$ With flavocytochrome $b_{2}$ and related enzymes that dehydrogenate $\alpha-\mathrm{OH}$ acids, a lysine $\varepsilon-\mathrm{NH}_{3}^{+}$located near the flavin $\mathrm{N}(1)$ brings about this stabilization. ${ }^{39}$ In pkDAAO an $\alpha$-helix dipole has been proposed to be involved in this stabilization. ${ }^{10}$ With RgDAAO, however, no arrangement of (partial) positive charges that could have a similar role is evident from the 3D structure. This thus raises the question about the mechanism of stabilization in RgDAAO: two cases can be differentiated. In the first, substrate dehydrogenation (equation (1)) contains the equilibrium of the two Michaelis complexes at the levels of oxidized and reduced enzyme as depicted in equation (4):

$$
\begin{aligned}
\{\mathrm{DAAO} & \left.\sim \mathrm{FAD}_{\mathrm{ox}} \sim \mathrm{R}-\mathrm{CH}\left(-\mathrm{NH}_{2}\right)-\mathrm{COO}^{-}\right\} \\
& \Leftrightarrow\left\{\mathrm{DAAO} \sim \mathrm{FAD}_{\mathrm{red}} \mathrm{H}^{-}\right. \\
& \left.\sim \mathrm{R}-\mathrm{C}\left(=\mathrm{NH}_{2}^{+}\right)-\mathrm{COO}^{-}\right\}
\end{aligned}
$$

(Note that a $\mathrm{H}^{+}$from the $\alpha$-amino group is released prior/concomitantly with the dehydrogenation, i.e. the " $\mathrm{NH}_{2}$ form" of bound amino acid is the one that reacts efficiently. ${ }^{12}$ )

In equation (4) the stabilization of negatively charged, reduced flavin is by the positively charged $\alpha$-imino $\left(\alpha=\mathrm{NH}_{2}^{+}\right)$product. ${ }^{12}$ The latter is positioned above the flavin pyrimidine ring and in an ideal position to stabilize its negative charge. ${ }^{12}$ The second case occurs when the positive charge associated with the ligand is absent: to account for stabilization we proposed that Arg285 plays a dual role. ${ }^{14}$ In the presence of a ligand having a carboxylic group it serves in its binding. In its absence, the guanidinium side-chain of Arg285 rotates around the $\mathrm{C}^{\varepsilon}$ bond to come in close proximity $(\sim 3 \AA)$ to the flavin pyrimidine ring and above its plane, where it can exert a neutralizing function. Modelling studies show that with pkDAAO the equivalent Arg283 can do the same (not shown). We would thus infer that also with pkDAAO the stabilization of negatively charged flavin species results primarily from the interaction with this Arg, whereby the invoked helix dipole ${ }^{10}$ can play an ancillary role.

Comparison of the structures of DAAO and $\mathrm{LAAO}^{17}$ demonstrates that the groups involved in binding of the substrate $\alpha \mathrm{COO}^{-}$and $\alpha \mathrm{NH}_{2}$ groups (interactions (a) and (b), see Results) form a mirror plane intersecting the axis formed by the substrate $\alpha \mathrm{C}-\mathrm{H}$ function and the flavin $\mathrm{N}(5)$ position. The discrimination by DAAO and LAAOs towards D and L-enantiomers can thus be rationalized in terms of a classical three-point attachment model (interactions (a), (b) and (c), above). This also would be equivalent to the four-location model recently discussed by Koshland: ${ }^{40}$ the fourth site being a direction, i.e. the orientation of the $\alpha \mathrm{C}-\mathrm{H}$ orbital with respect to flavin $\mathrm{N}(5)$ HOMO orbital. In LAAO and pkDAAO, the ligand amino group interacts with the carbonyl oxygen atom of a conserved glycine (Gly464 and Gly313, respectively), which is located at the same position as Ser335 in RgDAAO. Recently, the hydroxyl group of Ser335 has been proposed to play a role in transfer of a $\mathrm{H}^{+}$to bulk solvent, ${ }^{16}$ a function possibly carried out by His223 in LAAO. ${ }^{17}$ A further intriguing difference between DAAO and LAAO is the presence in the latter of a water molecule (W605) H-bonded to the flavin $\mathrm{N}(5)$ and to the side-chain of Lys326. ${ }^{17}$ It has been suggested that Lys326 is involved in hydrolysis of the imino product by Trp605. Clearly such a mechanism would not appear to be viable in $\operatorname{RgDAAO}$, due to the absence of analogous groups.

Some interesting similarities exist also with D-amino acid aminotransferase (D-AAT), a bacterial enzyme capable of transaminating D-amino acid residues and important for synthesis of D-glutamate and D-alanine, which are components of the bacterial cell wall. Also in this enzyme an extended loop (Gly94-Pro109) is important for the monomer-monomer contact (and provides residues to the cofactor-binding region and active site). ${ }^{41}$ Both DAAO and D-AAT recognize D-amino acid residues as substrates and possess a "carboxylate trap" (Arg285 in RgDAAO and Arg98 in D-AAT) and a "side-chain pocket" that discriminates substrate species. This cavity forms a pocket in which the side-chain of the substrate can be trapped and provides a surface for interaction with the side-chain.

In conclusion, comparison of the 3D structures of yeast and mammalian DAAO suggests that evolutive pressure has led to two enzymes that share the same chemical process, but use different kinetic mechanisms for catalysis. In the case of RgDAAO, and as a consequence of catabolic requirements, optimal catalytic efficiency has evolved leading to the (chemical) step of hydride transfer being ratelimiting. With mammalian DAAO we assume that the necessity to regulate activity has caused product release to be limiting. This diversity is 
Table 2. Data collection and refinement

\begin{tabular}{|c|c|}
\hline \multicolumn{2}{|l|}{ A. Diffraction data } \\
\hline $\begin{array}{l}\text { Redox state of flavin } \\
\text { Resolution }(\AA) \\
\text { Cell axes }(\AA) \\
\text { Observed reflections } \\
\text { Unique reflections } \\
\text { Completeness }(\%) \\
R_{\text {mgr }-\mathrm{F}} \\
I / \sigma\end{array}$ & $\begin{array}{c}\text { Oxidized } \\
100-1.9(2.0-1.9) \\
a=b=120.8 ; c=136.8 \\
193,503 \\
75,551 \\
99.0(99.8) \\
13.8(28.7) \\
5.3(1.9)\end{array}$ \\
\hline $\begin{array}{l}\text { B. Refinement } \\
R \text {-factor } / R \text {-free (\%) } \\
\text { No. of protein atoms } \\
\text { Solvent molecules with full occupancy } \\
\text { Solvent molecules with half occupancy } \\
\text { Substrates, ligands, and cofactor }\end{array}$ & $\begin{array}{c}20.5 / 24.8 \\
2799 \\
254 \\
118 \\
2 \text { anthranilates, FAD }\end{array}$ \\
\hline $\begin{array}{l}\text { Model r.m.s. deviations from ideality } \\
\text { Bond lengths }(\AA) \\
\text { Bond angles }\left(^{\circ}\right)\end{array}$ & $\begin{array}{c}0.018 \\
3.21\end{array}$ \\
\hline $\begin{array}{l}\text { Parentheses denote the highest shell. } \\
\text { a Calculations performed with SHEL }\end{array}$ & PRO. $^{44}$ \\
\hline
\end{tabular}

implemented by the use of different types of "lids" that cover the active site and are involved in uptake and release of ligand.

\section{Materials and Methods}

\section{Preparation of the protein and crystallization}

Wild-type RgDAAO was expressed and purified in Escherichia coli using the pT7-DAAO expression system in BL21(DE3)pLysS E. coli cells. The purified DAAO is stable in the $0.1-10 \mathrm{mg} / \mathrm{ml}$ protein concentration range and has spectral and kinetic properties identical with those of native enzyme. ${ }^{19}$ The purified protein was concentrated up to $10 \mathrm{mg} / \mathrm{ml}$ and equilibrated in $20 \mathrm{mM}$ Hepes buffer at $\mathrm{pH} 7.5$ by gel-permeation chromatography on a Sephadex G-25 (PD10) column. The dynamic light-scattering analysis was performed with model DynaPro 801 (Protein Solutions, Ltd). The recombinant form of $\mathrm{RgDAAO}$ was crystallized by vapour diffusion method at $20^{\circ} \mathrm{C}$, as reported. ${ }^{12}$ The crystals obtained by this method were found to contain L-lactate in variable amounts. The source of this ligand was found to be a lactate contamination $(\approx 0.4 \mathrm{~g} / \mathrm{kg})$ of commercial PEG1000 (FLUKA). The RgDAAO-anthranilate complex was obtained by soaking the crystals in the pure cryo-protection solution containing $30 \mathrm{mM}$ of the ligand. Binding was followed by observation of the crystal colour changes from yellow to reddish, this being complete within one minute.

\section{Solution of the RgDAAO crystal structure}

The anthranilate dataset was collected under cryogenic conditions. Prior to flash freezing, the crystals were transferred into the cryo-protectant solution, consistent with the reservoir solution and $20 \%(\mathrm{v} / \mathrm{v})$ glycerol. Data collection was on a rotating anode source (Schneider, Offenburg, Germany) using the MAR345 image plate system. Space group determination and data reduction was carried out in XDS. ${ }^{42}$ The refined model omitting L-lactate was the starting point for solving the structure of $\operatorname{RgDAAO}$ in anthranilate complex. The refinement employed a torsion angle dynamics $^{43}$ at a starting temperature of $5000 \mathrm{~K}$. Refinement was continued with SHELXL. ${ }^{44}$ The restraints were set in accordance with the resolution of the data and continuous control of the stereochemical quality of the structures was done with PROCHECK. ${ }^{45}$ For details of data collection statistics and refinement see Table 2 . Structure plots were produced with the programs MOLSCRIPT $^{46}$ and Raster3D ${ }^{47}$.

\section{Comparison of GR family members}

A superposition calculation was performed for each member of the $\mathrm{GR}_{2}$ family using the program Superimpose $^{48}$ and parameters describing the superposition were extracted with lsqman $^{49}$ from the best topological superposition.

\section{Accession numbers}

The coordinates and structure factors are deposited in the RCSB Protein Data Bank under accession code 1c0i.

\section{Acknowledgements}

This work was supported by a grant from Italian MIUR to M.S.P. (PRIN 2000-2002 Prot. MM05C73482). The CPU consuming calculations with a parallel version ${ }^{50}$ of SHELXL were performed on a SGI Origin 2000 at the University of Freiburg, Germany. We thank A. Brosig for preparing Figure 6.

\section{References}

1. Krebs, H. A. (1935). Metabolism of amino-acids. III. Deamination of amino-acids. Biochem. J. 29, 1620-1644.

2. La Rue, T. A. \& Spencer, J. F. T. (1967). The utilization of D-amino acids by yeasts. Can. J. Microbiol. 13, 777-788

3. Pilone, M. S., Verga, R., Fretta, A. \& Hanozet, G. M. (1989). Induction of D-amino acid oxidase by D-alanine in Rhodotorula gracilis grown in defined medium. J. Gen. Microbiol. 135, 593-600.

4. Pilone, M. S. (2000). D-Amino acid oxidase: new findings. Cell. Mol. Life Sci. 57, 1732-1747.

5. Schell, M. J., Molliver, M. E. \& Snyder, S. H. (1995). D-Serine, an endogenous synaptic modulator: localization to astrocytes and glutamate-stimulated release. Neurobiology, 92, 3948-3952.

6. Pilone, M. S. \& Pollegioni, L. (2002). D-Amino acid oxidase as an industrial biocatalyst. Biocatal. Biotransf. 20, 145-159.

7. Pollegioni, L., Langkau, B., Tischer, W., Ghisla, S. \& Pilone, M. S. (1993). Kinetic mechanism of D-amino acid oxidase from Rhodotorula gracilis and Trigonopsis variabilis. J. Biol. Chem. 268, 13850-13857.

8. Porter, D. J. T., Voet, J. G. \& Bright, H. J. (1977). Mechanistic features of the D-amino acid oxidase reaction studied by double stopped flow spectrophotometry. J. Biol. Chem. 252, 4464-4473. 
9. Curti, B., Ronchi, S. \& Pilone Simonetta, M. (1992). Dand L-amino acid oxidases. In Chemistry and Biochemistry of Flavoenzymes (Müller, F., ed.), vol. 3, pp. 69-94, CRC Press, Boca Raton.

10. Mattevi, A., Vanoni, M. A., Todone, F., Rizzi, M., Teplyakov, A., Coda, A. et al. (1996). Crystal structure of D-amino acid oxidase: a case of active site mirrorimage convergent evolution with flavocytochrome b. Proc. Natl Acad. Sci. USA, 93, 7496-7501.

11. Mizutani, H., Miyahara, I., Hirotsu, K., Nishina, Y., Shiga, K., Setoyama, C. \& Miura, R. (1996). Threedimensional structure of porcine kidney D-amino acid oxidase at $3.0 \AA$ resolution. J. Biochem. (Tokyo), 120, 14-17.

12. Umhau, S., Pollegioni, L., Molla, G., Diederichs, K., Welte, W., Pilone, M. S. \& Ghisla, S. (2000). The $\mathrm{X}$-ray structure of D-amino acid oxidase at very high resolution identifies the chemical mechanism of flavin-dependent substrate dehydrogenation. Proc. Natl Acad. Sci. USA, 97, 12463-12468.

13. Harris, C. M., Molla, G., Pilone, M. S. \& Pollegioni, L. (1999). Studies on the reaction mechanism of Rhodotorula gracilis D-amino acid oxidase: role of the highly conserved Tyr223 on substrate binding and catalysis. J. Biol. Chem. 274, 36235-36240.

14. Molla, G., Porrini, D., Job, V., Motteran, L., Vegezzi, C., Campaner, S. et al. (2000). Role of arginine 285 in the active site of Rhodotorula gracilis D-amino acid oxidase. J. Biol. Chem. 275, 24715-24721.

15. Pollegioni, L., Blodig, W. \& Ghisla, S. (1997). On the mechanism of D-amino acid oxidase. Structure/ linear free energy correlations and deuterium kinetic isotope effects using substituted phenylglycines. J. Biol. Chem. 272, 4924-4934.

16. Harris, C. M., Ghisla, S. \& Pollegioni, L. (2001). pH and kinetic effects in D-amino acid oxidase catalysis. Evidence for a concerted mechanism in substrate dehydrogenation via hydride transfer. Eur. J. Biochem. 268, 1-18.

17. Pawelek, P. D., Cheah, J., Coulombe, R., Macheroux, P., Ghisla, S. \& Vrielink, A. (2000). The structure of L-amino acid oxidase reveals the substrate trajectory into an enantiomerically conserved active site. EMBO J. 19, 4204-4215.

18. Miura, R., Setoyama, C., Nishina, Y., Shiga, K., Mizutani, H., Miyahara, I. \& Hirotsu, K. (1997). Structural and mechanistic studies on D-amino acid oxidasesubstrate complex: implications of the crystal structure of enzyme-substrate analog complex. J. Biochem. (Tokyo), 122, 825-829.

19. Molla, G., Vegezzi, C., Pilone, M. S. \& Pollegioni, L. (1998). Overexpression in Escherichia coli of a recombinant chimeric Rhodotorula gracilis D-amino acid oxidase. Prot. Express. Purif. 14, 289-294.

20. Faotto, L., Pollegioni, L., Ceciliani, F., Ronchi, S. \& Pilone, M. S. (1995). The primary structure of D-amino acid oxidase from Rhodotorula gracilis. Biotechnol. Letters, 17, 193-198.

21. Macheroux, P., Seth, O., Bollschweiler, C., Schwarz, M., Kurfürst, M., Au, L. C. \& Ghisla, S. (2001). L-amino-acid oxidase from the Malayan pit viper Calloselasma rhodostoma. Comparative sequence analysis and characterization of active and inactive forms of the enzyme. Eur. J. Biochem. 268, 1679-1686.

22. Rossmann, M. G., Moras, D. \& Olsen, K. W. (1974). Chemical and biological evolution of nucleotidebinding proteins. Nature, 250, 194-199.
23. Dyn, O. \& Eisenberg, D. (2001). Sequence-structure analysis of FAD-containing proteins. Protein Sci. 10, 1712-1728.

24. Campaner, S., Pollegioni, L., Ross, B. \& Pilone, M. S. (1998). Limited proteolysis and site-directed mutagenesis revealed the origin of microheterogeneity of Rhodotorula gracilis D-amino acid oxidase. Biochem. J. 330, 615-621.

25. Wierenga, R. K., Drenth, J. \& Schulz, G. E. (1983). Comparison of the three-dimensional protein and nucleotide structure of the FAD-binding domain of $p$-hydroxybenzoate hydroxylase with the FAD- as well as NADPH-binding domains of glutathione reductase. J. Mol. Biol. 167, 725-739.

26. Schulz, G. E. (1992). Binding of nucleotides by proteins. Curr. Opin. Struct. Biol. 2, 61-67.

27. Fraaije, M. W. \& Mattevi, A. (2000). Flavoenzymes: diverse catalysts with recurrent features. Trends Biochem. Sci. 25, 126-132.

28. Pollegioni, L., Ghisla, S. \& Pilone, M. S. (1992). Studies on the active center of Rhodotorula gracilis D-amino acid oxidase and comparison with pig kidney enzyme. Biochem. J. 286, 389-394.

29. Pilone, M. S., Pollegioni, L., Casalin, P., Curti, B. \& Ronchi, S. (1989). Properties of D-amino-acid oxidase from Rhodotorula gracilis. Eur. J. Biochem. 180, 199-204.

30. Casalin, P., Pollegioni, L., Curti, B. \& Pilone, M. S. (1991). A study on apoenzyme from Rhodotorula gracilis D-amino acid oxidase. Eur. J. Biochem. 197, 513-517.

31. Brünger, A. T., Adams, P. D., Clore, G. M., DeLano, W. L., Gros, P., Grosse-Kunstleve, R. W. et al. (1998). Crystallographic and NMR system: a new software suite for macromolecular structure determination. Acta Crystallog. sect. D, 54, 905-921.

32. Todone, F., Vanoni, M. A., Mozzarelli, A., Bolognesi, M., Coda, A., Curti, B. \& Mattevi, A. (1997). Active site plasticity in D-amino acid oxidase: a crystallographic analysis. Biochemistry, 36, 5853-5860.

33. Pollegioni, L., Fukui, K. \& Massey, V. (1994). Studies on the kinetic mechanism of pig kidney D-amino acid oxidase by site-directed mutagenesis of tyrosine 224 and tyrosine 228. J. Biol. Chem. 269, 31666-31673.

34. Pollegioni, L., Ceciliani, F., Curti, B., Ronchi, S. \& Pilone, M. S. (1995). Studies on the structural and functional aspects of Rhodotorula gracilis D-amino acid oxidase by limited proteolysis. Biochem. J. 310, 577-583.

35. Mattevi, A., Vanoni, M. A. \& Curti, B. (1997). Structure of D-amino acid oxidase: new insights from an old enzyme. Curr. Opin. Struct. Biol. 7, 804-810.

36. Benson, T. E., Filman, D. J., Walsh, C. T. \& Hogle, J. M. (1995). An enzyme-substrate complex involved in bacterial cell wall biosynthesis. Nature Struct. Biol. 2, 644-653.

37. Mecozzi, S., West, A. P., West, L. \& Dougherty, D. A. (1996). Cation-pi interactions in aromatics of biological and medicinal interest: electrostatic potential surfaces as a useful qualitative guide. Proc. Natl Acad. Sci. USA, 93, 10566-10571.

38. Edmondson, D. \& Ghisla, S. (1999). Flavoenzyme structure and function. Approaches using flavin analogues. Methods Mol. Biol. 131, 157-179.

39. Xia, Z.-X., Shamala, N., Bethge, P. H., Lim, L. W., Bellamy, H. D., Xuong, N. H. et al. (1987). Threedimensional structure of flavocytochrome $b_{2}$ from baker's yeast at $3.0 \AA$ resolution. Proc. Natl Acad. Sci. USA, 84, 2629-2633. 
40. Mesecar, A. D. \& Koshland, D. E., Jr (2000). A new model for protein stereospecificity. Nature, 403, 614-615.

41. Sugio, S., Petsko, G. A., Manning, J. M., Soda, T. \& Ringe, D. (1995). Crystal structure of a D-amino acid aminotransferase: how the protein controls stereoselectivity. Biochemistry, 34, 9661-9669.

42. Kabsch, W. J. (1988). Evaluation of single crystal X-ray diffraction from a position-sensitive detector. J. Appl. Crystallog. sect. A, 50, 157-163.

43. Brünger, A. T., Adams, P. D. \& Rice, L. M. (1997). New applications of simulated annealing in X-ray crystallography and solution NMR. Structure, 15, 325-336.

44. Sheldrick, G. M. \& Schneider, T. R. (1997). SHELXL: high-resolution refinement. Methods Enzymol. 277, 319-343.

45. Laskowski, R. A., MacArthur, M. W., Moss, D. S. \& Thornton, J. M. (1993). PROCHECK: a program to check the stereochemical quality of protein structures. J. Appl. Crystallog. 26, 283-291.
46. Kraulis, P. J. (1991). MOLSCRIPT: a program to produce both detailed and schematic plots of protein structures. J. Appl. Crystallog. 24, 946-950.

47. Merrit, E. A. \& Murphy, M. E. P. (1994). Raster3D version 2.0. A program for photorealistic molecular graphics. Acta Crystallog. sect. D, 50, 869-873.

48. Diederichs, K. (1995). Structural superposition of proteins with unknown alignment and detection of topological similarity using a six-dimensional search algorithm. Proteins: Struct. Funct. Genet. 23, 187-195.

49. Kleywegt, G. J. (1999). Experimental assessment of differences between related protein crystal structures. Acta Crystallog. sect. D, 55, 1857-1884.

50. Diederichs, K. (2000). Computing in macromolecular crystallography using a parallel architecture. J. Appl. Crystallog. 33, 1154-1161.

51. Diederichs, K. \& Karplus, P. A. (1997). Improved $R$-factors for diffraction data analysis in macromolecular crystallography. Nature Struct. Biol. 4, 269-275.

Edited by R. Huber

(Received 22 May 2002; received in revised form 17 September 2002; accepted 26 September 2002) 